\title{
El conflicto escolar desde las percepciones de jóvenes infractores en la escuela ${ }^{1}$ The school conflict from young offender's perceptions in school
}

\author{
John Alexander Usuga Oquendo ${ }^{2}$ \\ john.usugaoq@amigo.edu.co
}

\section{Resumen}

La investigación cualitativa centrada en la pregunta: ¿Qué percepciones tienen los jóvenes acerca del conflicto escolar y su resolución en las instituciones educativas? se sustenta en la metodología de análisis de caso aplicada en una institución que por su enfoque pedagógico orientado a la educación y la reeducación y por las particularidades de la población estudiantil ofrece posibilidades para la generación de conocimiento alrededor del conflicto como categoría central y objeto de estudio. La estrategia de recolección de información articuló grupos focales y entrevistas semiestructuradas. El proceso investigativo ofreció conclusiones como: el desconocimiento por parte de los adultos de la sutil diferencia entre violencia y conflicto, la importancia del rol del educador como figura decisiva en la prevención de este fenómeno y su intervención. Sobre la norma se concluyó el reconocimiento que le otorgan y su importancia para la regulación de la convivencia, pero simultáneamente se observó cómo la obligatoriedad de su cumplimiento es causal de conflicto, lo que denota gran ambivalencia en el momento de su aplicación.

Palabras clave: Conflicto escolar; violencia escolar; convivencia; autoridad.

\begin{abstract}
This qualitative research based on the question: what perceptions do youths have about the school conflict and its resolution within educational institutions? is based on case analysis methodology applied in a specific school due to its pedagogical approach on education and reeducation and its students' particularities offers the possibility to generate knowledge in conflict as a fundamental category and study object. The data collection tools were focus groups and semi-structured interviews. The research process offered high-value conclusions as the adults lack of knowledge about the subtle difference between violence and conflict, the relevance of the educator role for preventing conflict as well as his/her intervention. As conclusions, the recognition and importance of the law that regulates co-living are presented, as well as how the accomplishment of that law causes conflict, which denote ambiguity when applying it.
\end{abstract}

Keywords: School conflict; School violence; Co-living; Authority.

\footnotetext{
${ }^{1}$ Artículo de investigación.

${ }^{2}$ Teólogo y psicólogo. Docente de la Universidad Católica Luis Amigó. ORCID https://orcid.org/0000-0001-5605-7602

*Cómo citar este artículo: Usuga, J. (2018). El conflicto escolar desde las percepciones de jóvenes infractores en la escuela. Hojas y Hablas, (15), 84-99. DOI: 10.29151/ hojasyhablasn.15a5
} 


\section{Introducción}

La institución escolar no ha quedado al margen de los procesos de fragmentación, exclusión y desigualdad (Di Napoli, 2012) propios de las sociedades contemporáneas, por sus características como agente de socialización se le delega de manera acrítica y un tanto injusta, desde la familia y el estado, la tarea principal en la formación de la subjetividad social y política de los individuos $y$, en consecuencia, se le reclama sobre su responsabilidad en los actos $\mathrm{y}$ decisiones con los que éstos transgreden el orden anhelado o establecido; por ello se olvida que como constructora, pero igualmente como producto de una cultura, requiere una mirada sistemática dentro de su entramado de relaciones, ya que en ella se hacen evidentes las dinámicas y problemáticas de la sociedad en un nivel micro.

En el acontecer de la escuela, los diversos actores asumen roles de poder cargados de simbolismo, muchos de ellos observados $y$ aprendidos en la familia o bajo el influjo de otras instancias socializadoras que inciden determinantemente no sólo en la subjetividad sino también en las relaciones que operan como eje de la diferenciación/identificación entre los distintos grupos de estudiantes (Di Napoli, 2012) determinando formas de actuación, interacciones y vivencias complejas como la violencia y el conflicto.

Como categoría de referencia este último aparece reiteradamente con uso polisémico en la literatura derivada de la investigación en el espacio escolar, asociado usualmente a la violencia escolar pero también al acoso escolar, el maltrato, el bullying e incluso a la indisciplina, además es posible ubicarlo en las diferentes referencias con alusiones a las violencias y las microviolencias. No obstante, el conflicto escolar también llega a entenderse como una categoría específica y un tanto concreta, si se compara con la violencia escolar ya que aquella incluye para su comprensión a otras más particulares como el acoso escolar (Olweus, 2004) o la indisciplina.

Esta multiplicidad y dispersión en su uso genera dificultades incluso para estructurar un referente teórico específico, además porque el estado de la investigación sobre ambos fenómenos revela un amplio repertorio de estudios, objetos y elaboraciones sobre la violencia escolar, a diferencia de un horizonte aún restringido en relación con el conflicto en dicho contexto.

Si bien, conflicto y violencia aluden a fenómenos relacionales y se derivan de ellos, su comprensión y tratamiento como constructos teóricos, al igual que su intervención en la vida cotidiana son confusos en tanto uno y otro se asemejan en su carácter de hecho social o en términos de Castillo (2011) como "acontecimiento subjetivo de un nosotros" (p. 421) con un sustrato determinado por características culturales, del grupo y particulares de los individuos.

Desde esta perspectiva, y aunque aparentemente guardan similitudes que conducen a veces a confundirlos, no pueden entenderse de igual manera. La violencia, en la mayoría de los casos en la escuela, es consecuencia de un conflicto que no se ha resuelto, se trata de una discrepancia en los intereses de los implicados unida a la certeza de que sus expectativas o aspiraciones al respecto no podrán alcanzarse simultáneamente (Luna Bernal y De Gente-Casas, 2017), lo que ocasiona usualmente un problema, la violencia comúnmente se deriva de la no resolución del conflicto por medios pacíficos (Concha Eastman y Krug, 2002). 
Es fundamental la atención a todo tipo de alteraciones en el orden relacional del aula, ya que son justamente las situaciones poco trascendentales en apariencia y de baja intensidad pero no resueltas las más frecuentes causas del origen de la violencia (Di Napoli, 2012); estas, que en apariencia sólo rompen el clima cotidiano de clase y no desembocan en sus primeros momentos en lesiones físicas o síquicas contra profesores o estudiantes (Chaux, 2002) se tornan en factores de alto riesgo para el deterioro de la convivencia.

Pareciera que la diferencia entre violencia y conflicto estén asociadas a la intencionalidad de generar daño, el cual es deliberado en el acto violento pero es probable que no lo sea en la situación de conflicto. En esta investigación se asumen violencia y conflicto como dos categorías diferentes con profunda relación: no todo acto violento implica un conflicto, pero una situación de conflicto puede derivar en violencia.

Por tratarse de fenómeno sociales es posible identificar factores protectores frente a la violencia y el conflicto en los centros educativos, uno de estos es el clima escolar que se entiende como "el contexto o características psicosociales en que se dan las relaciones interpersonales" (Tijmes, 2012, p. 106) y cuyo elemento central, en torno al que se erigen $y$ fortalecen, son precisamente estas relaciones. El clima se deriva y se construye en el marco de la cultura escolar, es decir en la articulación de signos, significados, valores e imaginarios derivados de elementos prescritos, practicados, ocultos, hechos vida en las formas de relación. Por ello cuidar el clima es cuidar las circunstancias que atenúan, potencian o desencadenan conflictos, los que a su vez pueden detonar en formas de violencia (Castillo, 2011).

Otro factor protector tiene que ver con la valoración y el respeto a la diversidad. El empeño de la escuela por formar jóvenes iguales en sus características sociales, académicas y de intereses, entre otras, desconociendo su identidad en relación con sus contextos de origen y de socialización, es ya una causa para el origen del conflicto por cuanto se desconoce lo particular del otro (Di Napoli, 2012).

En este sentido es igualmente valiosa la atención al conflicto en sí mismo, a su esencia, origen y tratamiento, y a las inmensas posibilidades de resolverlo si desde la primera infancia los niños reciben entrenamiento para su afrontamiento (Chaux, 2002), también de aprovecharlo en pos del desarrollo social y político de los estudiantes y de la generación de entornos en y para la paz, esto en contravía del gran énfasis normativo otorgado en el sistema educativo colombiano a los manuales de convivencia, y en muchos de los casos también como recurso para lo punitivo, con lo que se menosprecia el afrontamiento en primera instancia del conflicto como otro factor para la prevención.

A partir de la Ley 1620 (MEN, 2013) y de su Decreto Reglamentario 1965 (MEN, 2013), el conflicto escolar como problemática objeto de estudio en el contexto colombiano reclama mayores y sistemáticas elaboraciones investigativas, generadoras de un marco conceptual más extenso que permita soportar diferencias y relaciones entre violencia y conflicto y los términos afines, pero que igualmente ofrezcan un nuevo panorama integral para dilucidar supuestos que circulan en los escenarios educativos, como por ejemplo el conflicto supeditado únicamente a las dificultades de convivencia propias de población de jóvenes adolescentes violentos (Di Napoli, 2012) o la clasificación de situaciones de conflicto como situaciones de violencia. 
El proyecto El conflicto escolar desde las comprensiones de jóvenes infractores de la ley en una I.E. orientada a la educación - reeducación tiene su génesis en los intereses de la Maestría en Educación de la Universidad Católica Luis Amigó en su propósito de abordar una problemática que por las situaciones de conflicto y violencia en varios territorios de Colombia y por su alto impacto en los distintos espacios sociales y simbólicos se tornó paulatinamente lugar común para la generación de interrogantes inherentes a los procesos educativos.

El objetivo general propuesto, derivado del proyecto antes anotado, y que se ha constituido en la pauta orientadora para el trabajo es comprender las construcciones que los jóvenes tienen sobre el conflicto escolar a partir de sus vivencias en una I. E. orientada a la educación y la reeducación. El telón de fondo que lo motivó se sustenta en la realidad del país, ya que sus dinámicas sociopolíticas en los últimos años han tenido un sello fundamental centrado en el conflicto y en su resolución, esto debido a la búsqueda de la paz después de más de 60 años de guerra, situación que obligatoriamente ha permeado los escenarios educativos y las dinámicas en los espacios escolares, de igual manera se constituyen en pretexto para este cometido las investigaciones sobre temáticas afines al conflicto sobre las que se observa como "de manera reiterada los estudios resaltan el impacto de problemas asociados a la agresividad y la violencia presentes en la escuela como problemas sociales en constante crecimiento" (Ramírez y Arcila, 2013, p. 419).

Con estas como preámbulo se dio lugar a una búsqueda donde se visibilizarán las construcciones que los jóvenes han hecho desde sus vivencias en relación a los matices con que el fenómeno del conflicto se hace presente, en perspectiva de aportar nuevo conocimiento a las comunidades académicas y educativas.

Se acudió como guía epistemológica a una postura comprensiva, que en la investigación abre una oportunidad para generar nuevo conocimiento desde la descripción y la interpretación del conflicto y a su resolución en una institución que, precisamente por su población docente y en formación y por la experiencia en gestión, posee una amplio bagaje y un saber acumulado de alto valor, con lo que se ofrecería información a las comunidades académicas y en las instituciones educativas en general, ya que el conflicto como fenómeno social está inmerso en todos los espacios de interacción.

Desde la pregunta ¿Qué construcciones tienen los jóvenes acerca del conflicto escolar y su resolución en las instituciones educativas? se gestaron como objetivos específicos: 1) identificar las representaciones que los jóvenes han configurado acerca del conflicto escolar en su relación con la norma como decisión institucional para resolver el conflicto; 2) caracterizar los roles de los sujetos que intervienen en el conflicto escolar desde la experiencia de los jóvenes en sus procesos formativos; y 3) describir las formas de intervención de docentes y estudiantes ante las situaciones de conflicto escolar.

\section{Método}

Como estrategias de recolección de información se acudió en primera instancia a la aplicación de entrevistas semiestructuradas a once estudiantes de la institución seleccionada como caso, y posteriormente a la realización de grupos focales con el propósito de validar los análisis preliminares realizados sobre las entrevistas, pero además con el ánimo de ampliar la información. 
Se partió de las categorías iniciales de conflicto, resolución de este y violencia, todas en ellas en el contexto de una IE.

La opción por los once participantes obedeció al criterio de caso típico o muestreo por expertos como explica Rincón y Barreto (2016), fueron tres docentes conocedores de la población estudiantil de la IE quienes por su experiencia, saber y trayectoria en este centro indicaron los actores representativos entre los estudiantes, tuvieron en cuenta el tiempo de permanencia en la institución, su proceso de reeducación, sus avances académicos y sus personalidades extravertidas y dispuestas a aportar información de su experiencia de vida.

Los jóvenes hacen parte del Sistema de Responsabilidad Penal para Adolescentes (SRPA) establecido en Colombia por el Código de Infancia y Adolescencia (Ley 1098 de 2006); este es un sistema que permite, en primer lugar, administrar justicia a adolescentes en conflicto con la ley penal colombiana; en segundo lugar, tiene como finalidad proteger, educar y restaurar el proyecto de vida de estos jóvenes de tal manera que después del proceso puedan integrarse adecuadamente a la sociedad civil. El SRPA propone medidas no privativas de la libertad y medidas privativas de la libertad según el tipo de delito cometido, los 11 jóvenes hacen parte de las segundas, por ello todos están internos en un Centro de Atención Especializada (CAE), además todos asisten a una IE ubicada al interior de la planta física de la institución donde están cursando la media académica.

La aplicación de consentimientos informados se llevó a cabo después de su previa revisión por la funcionaria de Bienestar Familiar encargada y del rector de la institución, luego en una jornada de socialización al grupo muestra se presentó la propuesta investigativa en general y se explicaron los derechos y posibilidades de no participar en el proceso o de retirarse del mismo en cualquier momento.

Para la recolección de información se diseñó como instrumento orientador un guion de encuesta semi-estructurada y otro para grupos focales. En todo el proceso se acudió al uso de grabaciones.

Los datos globales recogidos se consideraron desde su cantidad y saturación informativa, con lo que se tuvo certeza de su suficiencia y de su adecuación, y con ello de su posibilidad para responder a los planteamientos y demandas del problema y de los objetivos.

Para el análisis preliminar y después de un proceso de selección y reducción sobre la información derivada de las entrevistas, se acudió a su consolidación en matrices analíticas sobre las categorías generadoras, con las afirmaciones y observaciones plasmadas en ellas se procedió a la revisión y agrupación de textos en perspectiva de develar categorías emergentes. El producto obtenido se constituyó en el material para la validación por parte de los grupos focales, los que ratificaron, nutrieron $y$ ofrecieron nuevas afirmaciones sobre las que se llevó a cabo un segundo ejercicio de análisis. Las conclusiones se generaron a partir de poner en diálogo las comprensiones derivadas de ambos momentos.

\section{Metodología}

Esta investigación se soportó en el paradigma cualitativo que considera el objeto de conocimiento y la realidad fundada en torno a este como "proceso histórico de construcción a partir 
de la lógica de diversos actores sociales" (Galeano, 2004, p. 46), lo que implicó la atención a miradas, razonamientos y análisis inscritos en la subjetividad e intersubjetividad, con todo lo que ello exige no sólo por la complejidad desde la cual deben entenderse, sino además por la exigencia de rigurosidad que se asumió como imperativo y como atributo fundante de la pesquisa, desde la problematización hasta la generación de hallazgos y conclusiones.

Epistemológicamente se asumieron fundamentos complementarios: el enfoque hermenéutico y la orientación construccionista. Desde el primero, la acción fue siempre dinámica y de permanente interpretación y búsqueda de la comprensión sobre el material de análisis recolectado; la segunda, exigió en todo momento la "atención hacia el mundo de la intersubjetividad compartida, y la construcción social del significado y del conocimiento" (Sandín, 2003, p. 174). Ambos, interpretación y construcción, fueron germen para un ejercicio que metafóricamente puede ilustrarse como una espiral, que al avanzar se nutre de las elaboraciones anteriores acogiendo el todo, para recrearlo y nutrirlo permanentemente de sentidos y significados.

Metodológicamente, y en armonía con lo planteado, se optó por el estudio de caso situacional que "estudia un acontecimiento, desde la perspectiva de los que han participado en el mismo" (Rodríguez, Gil y García, 1996, p. 96) como estrategia para la comprensión de fenómenos sociales de interés particular, en el cual se aborda un caso o "varios casos, que combinan diferentes métodos para la recolección de la evidencia" (Páramo, 2016, p. 309).

Entendiendo con Míguez (2017) que el análisis de caso no habilita a hacer generalizaciones, se acogió esta metodología ya que en las posibilidades que ofrece para "el examen detallado, comprehensivo, sistemático y en profundidad del caso objeto de estudio" (Sandín, 2003, p. 175) permite atender a los requisitos de un fenómeno con particularidades de alto interés para la generación de nuevo conocimiento.

La selección del caso fue en una institución de carácter educativo - reeducativo dirigida por religiosos dedicados a la formación de población masculina con problemáticas de socialización, obedeció a su extensa trayectoria en situaciones de conflicto y violencia suscitadas en los aconteceres cotidianos entre estudiantes y docentes, pero además a su gran legitimidad local y nacional para promover procesos para la restitución de los derechos de niños, adolescentes y jóvenes a partir de una profunda confianza en sus capacidades particulares para recuperarse, rescatar su lugar en la sociedad y ser co-creadores de nuevas y mejores situaciones vitales, todo ello en una constante apuesta por la recuperación del joven, a su convicción en el acompañamiento en la misericordia y en la posibilidad de una educación con sentido realista del ser humano.

\section{Resultados y discusión}

Los resultados obtenidos en esta investigación respecto al conflicto escolar en perspectiva de su conocimiento desde la mirada de los actores que lo han vivido, en este caso los jóvenes de una institución educativa centrada en la educación y la reeducación, nacen del análisis sobre sus construcciones personales acerca del origen de este fenómeno, significado, relaciones y vivencia; en consecuencia son producto de las tramas de significación instituidas por ellos en lo cotidiano, erigidas en sus contextos de actuación y en las interacciones que estos proveen para configurar 
nuevos y permanentes sentidos, por ello en esta ruta de comprensión se asumió como punto de partida para la definición de la categoría generadora, en la voz de los participantes, que el conflicto debe entenderse como situación que antecede la violencia y que surge de una inconformidad o un desacuerdo consigo mismo o con otro, "yo digo que conflicto es el inicio del problema y violencia es la solución" (joven c), o que la "violencia es como ya pasar del conflicto al acto, de un... como de una discusión a una agresión" (joven g).

Esta alusión de alta recurrencia entre los entrevistados permitió afirmar que hay diferencias entre ambos fenómenos y entre sus vivencias, es decir entre el conflicto y la violencia, pero a pesar de ello sus límites son difíciles de marcar en un sinnúmero de situaciones ya que bastan "expresiones o acciones propias de un momento de impulsividad, de pérdida de la calma y de no reflexionarse" (joven d) para que el conflicto pase a ser violencia, son los ataques verbales o físicos los que marcan la diferencia.

No obstante para todos los jóvenes que participaron en el proceso el conflicto puede ser benéfico cuando en su acontecer no se suscitan acciones de agresión, ya que lo entienden como vivencia de orden cotidiano, propia de las interacciones en cualquier grupo social, que ofrece amplias posibilidades a puntos de vista contrarios que dan cuenta de la diversidad de los sujetos y de sus múltiples y diferentes miradas del mundo, por ello ratifican en el mismo una valiosa oportunidad para la construcción social como también lo expresa Luna Bernal y De Gente-Casas (2017), así también Salinas, Posada e Isaza (2016) lo conciben como impulso natural y respuesta positiva de la condición humana.

Sin embargo esta connotación cambia cuando no se resuelve tan pronto surge o se hace evidente, ya que se transforma en violencia; por ello los jóvenes son claros en anotar que cualquier situación conflictiva, bien sea escolar o en otros entornos, no puede descuidarse sino que debe atenderse e intervenirse inmediatamente, lo que en su forma de expresión implica una 'intervención de choque' porque de lo contrario puede generarse una situación de mayor riesgo e impacto, de caos o 'revolución' en la que finalmente se alteran los ánimos de todos, el grupo toma parte y se incrementan los sentimientos de malestar e incluso de ira de los implicados directamente, "he percibido que cuando se crea un conflicto entre dos personas ya la dinámica cambia, la intervención tiene que ser como más como de choque, porque, sí me entiende, ya todo se forma como una revolución" (joven i). Según Salinas et al. (2016) desatender el conflicto es incitar a la agresión y con ella a la violencia, desconocerlo y silenciarlo es ponerlo fácilmente al servicio de la destrucción y la violencia.

Respecto a los límites en la dupla conflicto/ violencia, su demarcación es difícil para los jóvenes, lo que para algunos puede significar un acto de violencia, para otros es muestra de la latencia de un conflicto o simplemente una manifestación brusca o grotesca usual en las interacciones, no obstante ellos aseveran que "el conflicto es un problema que se ve muchísimo, ¡eh! en circunstancias o situaciones negativas y puede llevar a demasiadas cosas, (...) a partir de un conflicto se da la violencia” (joven b), coinciden con Coronel y Puig de Stubrin (2013) en que:

Las manifestaciones más graves y espectaculares de la violencia no son las que más asiduamente ocurren en las comunidades escolares, mientras que las formas más habituales y cotidianas de conflicto (la trasgresión, las incivilidades, etc.) son las que tienen mayor incidencia. (p.151) 
Es probable que surja la violencia sin previas situaciones de conflicto hacia el otro; esto manifiesta que, en ocasiones, puede haber violencia sin que haya conflicto, pero para los jóvenes esta no es la ruta habitual en la diada conflicto/violencia en el escenario escolar. Al aludir a las consecuencias del conflicto, quizás lo más habitual es el ambiente pesado en el que predomina la rabia o el resentimiento contra el otro.

Un contexto diferente remite al consumo de sustancias psicoactivas como generador de violencia en diferentes escenarios y situaciones:

Desde mi punto de vista (...) por el consumo de sustancias sicoactivas se da una violencia, porque ya la sustancia psicoactiva te cambia a vos como, el estado de ánimo, también por la falta de tolerancia, por el machismo que tiene uno pues (...) hacer valer su opinión. (joven g)

Es importante enfatizar que este sentimiento puede ser una de las causas más fuertemente relacionadas con el origen del fenómeno y sobre la cual se sustenta su prolongación, con lo que se entiende que usualmente el conflicto se posterga en el tiempo y en las circunstancias pasando desapercibido para aquellos que no son sus protagonistas.

Pero las dinámicas se alteran cuando los compañeros de clase lo advierten, con lo cual el clima se afecta, los muchachos se sienten involucrados bien sea desde la emoción cifrada en la incomodidad o el malestar, pero también desde el impulso por tomar parte en el asunto para apoyar a alguno de sus pares, o para contribuir a superarlo, como sucede con quienes tienen roles de liderazgo.

También es consecuencia del conflicto la distracción ante las diversas actividades académicas, lo que incide en el cumplimiento de responsabilidades y el rendimiento en general, particularmente de quien se entiende como la víctima, quien se ocupará de pensar estrategias para liberarse de la situación y adelantarse a su opositor, o experimentará profundo temor, aún cuando sus posibles acciones ante el grupo denoten total valentía y decisión.

Acerca de las dinámicas que son propias en la situación de conflicto, fue de alta relevancia el énfasis puesto por los jóvenes en su coexistencia en las relaciones entre pares estudiantes, por lo que dejan de lado, por lo menos en el tratamiento espontáneo de la temática, lo que pueda suscitarse entre estudiantes y docentes/directivos o exclusivamente entre estos y otros actores diferentes a la institución. Su nivel de identificación y emotividad al respecto fue notorio, sus palabras y actitudes pusieron de manifiesto gran sensibilidad e inquietud.

La insinuación al conflicto con los profesores fue diferente, solo se evidenció al tratar la relación norma/conflicto:

Aprendí de ellos, la serenidad, no dejarme llevar de mis impulsos, controlar los impulsos, tener auto control de mí y ser consciente sobre las consecuencias que me puedan generar, (...) hacer lo positivo, lo que mejor se pueda hacer en una situación, siempre con la escucha, (...) con la necesidad de hacer lo correcto, entonces ahí hablamos de la justicia, o sea que ser justo es hacer lo correcto, lo positivo. (joven $\mathrm{f}$ )

Percibiendo a la mayoría de maestros como gran ayuda en la resolución no violenta del conflicto.

En el conflicto los estudiantes evidencian diversos roles: agredido y agresor, víctima y victimario, como ellos los denominan, de igual manera 
también hay otros alrededor que se vinculan porque se identifican con una de esas dos partes. Los jóvenes se refieren específicamente a esta situación como "pararse por el amigo", así "en términos callejeros yo me paro por mi amigo, yo pa' las que sea con él, yo no lo voy a dejar sólo, vamos a peliar si es necesario" (joven a).

En palabras de los chicos, esta forma de vincularse se constituye en un poderoso refuerzo para motivar y agrandar la situación haciéndola mayor y más extensa en el tiempo, pero además porque incita a premeditar acciones y usualmente agresiones en contra de los otros, lo que puede determinar la consolidación de un conflicto de mayores riesgos o la generación de actos violentos.

Otros roles, diferentes a los de los protagonistas, con motivaciones o participaciones directas también se hacen evidentes e incluyen a aquellos que buscan ser intermediarios para aportar a la resolución del conflicto mediante la invitación al diálogo, patrón que en la mayoría de las veces es asumido por otros estudiantes y que se corresponde con tres intencionalidades diferentes: la del "conciliador" -como ellos lo nombran-que opera en la convicción de la búsqueda del bien común y la resolución del conflicto como vía para la armonía entre compañeros, otra intención es la del interés de no verse afectados posteriormente como grupo ya que los docentes pueden generar sanciones grupales. Se trata en este caso del "beneficio propio", el colaborar para evitar un problema de otros con consecuencias comunes.

La lealtad toma forma mediante la decisión por contribuir con la resolución de la situación evitando con ello que el parcero salga lastimado, "uno ya sabe resolver los conflictos porque va cogiendo la experiencia, porque cuando uno ya tiene experiencia en un conflicto, uno siempre va tener la capacidad de resolver ese conflicto" (joven c).

Se ponen en evidencia lealtades que operan en vía a la resolución del conflicto, pero otras orientadas al incremento de su gravedad, Di Napoli (2012) alude a la toma de posiciones y decisiones en el marco de una red de interdependencias en las que "el sujeto no actúa solo a través de su individualidad sino que también se enmarca en distintos grupos de pertenencia con los cuales se siente identificado" (p. 30) y teje diversos vínculos mediados, por ejemplo, por el cariño, la solidaridad o la lealtad.

Sobre la intervención de quienes lo hacen en perspectiva del bien común, son altamente valorados algunos pares que logran tener gran habilidad para incidir proactivamente en el cese de las confrontaciones, por lo que son reconocidos y respetados, además gozan de legitimidad para llamar al diálogo a compañeros que detectan inmersos o a las puertas de iniciar un conflicto.

Como mediadores también aparecen ocasionalmente los educadores, pero sus posturas son cuestionadas por los encuestados, desde su percepción tal intervención obedece en primera instancia a la obligatoriedad de cumplir con una tarea, como las del contrato laboral, por ello la evaden cuando es posible. Con esto, los jóvenes concluyen la falta de convicción de algunos docentes por aportar constructiva y formativamente a esta situación y su decisión por no verse implicados en situaciones inherentes al conflicto entre estudiantes.

Lo anterior no invisibiliza la acción deliberada de los verdaderos educadores, pocos según los jóvenes, que, con su intervención inmediata, con criterio y convicción, a veces también con afecto hacia sus estudiantes, aportan para mediar 
ante un conflicto, para contener la situación y para hacerle seguimiento y tratamiento en el deseo de superarlo desde sus bases y en su totalidad.

Con participación de alta incidencia surgen también quienes con sus gestos o palabras incitan a incrementar la discordia o a la violencia. Al parecer, estos actores disfrutan de la situación como si fuese un espectáculo, por ello se esmeran por reforzar lo dicho en tono de agresión o por invitar a resolver el conflicto mediante los golpes. En esta vía, es notoria la insistencia de los chicos en aludir al desempeño, un tanto desalentador para ellos, de los docentes que ponen de manifiesto la intención de que los jóvenes por su cuenta, con sus recursos $\mathrm{y}$ decisiones sean quienes los resuelvan, evadiendo con ello cualquier compromiso lógico y propio de un educador, y validando cualquier opción asumida por los sujetos en conflicto.

El descuido deliberado por parte del educador es leído por sus estudiantes como incapacidad o apatía, ellos esperan su intervención y manifiestan que desde su rol tenga la capacidad de saber más sobre la situación con el propósito de hacer mucho más sobre ella:

A veces el educador está más pendiente de cortar y pegar informes y eso cuestiona mucho, ellos no tienen sentido de pertenencia, hacen lo que tienen que hacer y ya, ese mismo educador ve que hay un conflicto y no hace lo posible por evitar lo grave, sólo llegan a intervenir cuando se sacan sangre, pero otros [maestros] tienen sentido de pertenencia, pero no sólo por la casa sino también por las personas, por eso ayudan con los conflictos. (joven b)

El educador está llamado a intervenir ahí, bien sea porque logre aportar a subsanar la situación o no, está investido de una autoridad que le delega la institución y le confían la familia y la sociedad, es parte de sus obligaciones y de su compromiso y por lo tanto es una responsabilidad indelegable e impostergable; obligación, autoridad e interés por la contribución deben primar y complementarse para decidirse a actuar desde las configuraciones que hacen los muchachos.

También suele suceder que los educadores estén inmersos en otras tareas institucionales, a veces altamente demandantes desde la percepción de sus estudiantes, por lo cual descuidan la mirada a situaciones que van más allá de lo propio de los procesos de enseñanza/aprendizaje, en este caso a la convivencia, los códigos ocultos en las interacciones, lo explícito y lo implícito en las dinámicas cotidianas en sus grupos; son precisamente los momentos de descuido como estos, a los que los muchachos acuden para solucionar de manera particular sus divergencias mediante acciones que incrementan la gravedad del conflicto, "entonces yo le dije vámonos para el baño y allá solucionamos ese problema, y sí, en el baño se presenta la agresión para supuestamente solucionar el problema" (joven e).

Sin embargo una situación aparentemente contradictoria se pone de manifiesto ya que como se anotó, aunque esperan la presencia activa del docente, su rol como autoridad es puesto en cuestión e incluso rechazado permanentemente, debido a que para muchos de estos jóvenes que han vivido en situación de calle o que han estado adscritos a barras o combos al margen de la ley en la ciudad, la potestad de esta figura ha estado en manos de otros jóvenes, por lo que sus vivencias han determinado decisivamente construcciones personales acerca de la misma, con lo que les resulta difícil aceptar a otro diferente a su par; se trata de su "adhesión al orden social establecido" (López et al., 2011, p. 8), al que muchos de estos jóvenes han considerado determinante para su actuar y sus 
decisiones de relación con el mundo, "cuando uno ingresa a una institución como está a uno le dicen que el maestro es la autoridad, pero para uno no es así, porque no estamos adaptados a que un adulto nos llame la atención" (joven a).

La intromisión del adulto, en ocasiones, suscita no sólo el rechazo sino incluso 'el contrapunteo' posterior en lo cotidiano de las interacciones, máxime si la acción del educador está acompañada de expresiones que incitan a la amenaza o el temor, en ello los jóvenes coinciden en afirmar que es más fácil y válido seguir el consejo de sus compañeros, pues los interpretan como ayuda de pares y colegaje, y pueden ser totalmente tolerantes a sus intervenciones, ante las que no guardan resentimientos, lo que sí sucede con los educadores:

Y empieza el adulto a intervenir la situación, uno, el residente, se pone eufórico con más ira y pasa de tener el conflicto con el residente para tenerlo con la autoridad. Es como la falta de autoridad adulta que nunca tuvimos afuera, y desde lo que lleva a un conflicto, con la experiencia que hemos tenido en la calle uno es el que debe intervenir el conflicto, y más fácil. (joven $b$ )

Al hablar de la norma y su relación con el conflicto en el espacio escolar, los jóvenes afirman que es algo que usualmente no se comprende en primera instancia, pero que se aprende en la experiencia, en el día a día, en las dificultades para cumplirla y en los retos que impone, en la reflexión sobre las incoherencias que se suceden en torno a su cumplimiento, con todo ello paulatinamente se toma conciencia y se construye su noción como instrumento de alto valor para contribuir a la convivencia y al bienestar de las relaciones.

Aún a pesar de confrontarlas o aceptarlas a disgusto, las normas de lo cotidiano, las reguladoras de cualquier tipo de interacción social, pero también las normas declaradas institucionalmente generan, según los chicos, aprendizajes y forman actitudes que serán necesarias en los ámbitos de desempeño futuros, bien sean en espacios sociales o en ambientes laborales. De esta manera, la norma implica la puesta en acción de principios y de la ética de cada sujeto y su vulneración implica consecuencias no sólo para el sujeto que la quebranta, sino también para el colectivo en general, "yo diría que sin norma esto sería un caos, la vida se trata de normas, de hábitos, este mundo sin la norma no sería mundo" (joven $\mathrm{h}$ ), además "yo pienso si uno no cumple una simple norma entonces uno como va solucionar un conflicto más adelante, ¡cierto!" (joven i).

Las normas de los manuales de convivencia, desde la perspectiva de estos jóvenes, tienen valor, además porque para su construcción se designaron personas legítimas institucionalmente, sujetos con el conocimiento y la capacidad de entender y definir pautas necesarias y válidas para la convivencia, y ello tiene lugar no sólo en el contexto de una IE sino en cualquier espacio de la ciudad y de la sociedad en general.

La existencia de la norma puede determinar también decisiones para responder ante las situaciones de conflicto ya que la norma determina tácitamente las respuestas en situaciones de confrontación, condiciona y evita decisiones precipitadas. El temor a ser 'echado' de la institución o el de no ser recibido nuevamente en la institución reprime su transgresión, pero también el conflicto.

No obstante estas convicciones, los jóvenes son contradictorios al tratar de vislumbrar la relación entre el valor de la norma y su cumplimiento permanente ya que afirman tener conocimientos sobre ella y reiteran su valor, son opuestamente deliberados para acatarla en múltiples circunstancias. Es evidente que cumplen con facilidad 
aquellas que les agradan o no les deparan grandes restricciones, al contrario de las que suscitan pereza o desagrado o aquellas con las que advierten que pueden confrontar y despertar malestares en las figuras de autoridad, en este caso los docentes, como sus primeros portadores. Ir en contra de la norma, es esencialmente ir en contra de los demás, es la posibilidad de afectar al otro, de manifestarle su desacuerdo con él por lo que representa:

La única norma que nos gusta es a la que nosotros nos acomodamos siempre, la que nos gusta, la que nos relaja, la que nos es más fácil de cumplir, nosotros no somos capaz de cumplir una norma que nos exigen y que quizás más adelante un mañana nos va a hacer más humanos de lo que somos hoy. (joven $\mathrm{f}$ )

Resulta de alto valor, respecto a la consecuencia ante la vulneración de la norma, descubrir que más allá de lo punitivo los chicos defienden su propia identidad en primera instancia, por lo que son capaces de esperar incluso la peor sanción si consideran que la pauta legal se instituye en contra de su ser, de sus principios personales y de la aceptación que merecen de sus mayores, por ello la "sanción normalizadora" (Foucault, 2002, p. 158) negativa para algunos, representa para otros la ratificación de su dignidad y una manifestación ante la represión, "si mi identidad es tener la camisa afuera, entonces uno dice no hay consecuencia porque yo no estoy en una entrevista, o en una reunión o en algo así, entonces pienso que nosotros estamos sometidos, obligados" (joven h).

\section{Conclusiones}

En el campo educativo existe un terreno poco explorado concerniente al ámbito del conflicto y a su pervivencia como fenómeno diferente a la violencia escolar, esto a pesar de la promulgación en Colombia de normativas y políticas como la Ley 1620 (MEN, 2013) y su Decreto Reglamentario 1965 (MEN, 2013) del mismo año e incluso la Ley 1098 o Código de Infancia y Adolescencia (Congreso de la República, 2006), que ofrecen marcos de comprensión para generar nuevas y pertinentes estrategias sobre la protección integral, la prevalencia del interés superior, la intervención del conflicto y la promoción de la convivencia entre otros, además de dar orientaciones para disminuir o suprimir las situaciones de violencia.

En contraposición a lo anterior y en torno a la pregunta central de esta investigación: ¿Qué construcciones tienen los jóvenes acerca del conflicto escolar y su resolución en las instituciones educativas? ellos manifiestan interesantes elaboraciones sobre el fenómeno, entre ellas la clara diferencia entre los fenómenos conflicto y violencia en la IE, destacando lo natural y propio del primero en las interacciones humanas tal como lo plantean Coronel y Puig de Stubrin (2013) y la importancia de atenderlo tan pronto se gesta de manera que sea un factor de crecimiento de la subjetividad social, con lo que además se limitan posibilidades a la gestación de la violencia como fenómeno subsidiario al conflicto desatendido Concha-Eastman y Krug (2002). En consecuencia, mirarlo oportunamente e intervenirlo en su real magnitud se constituye en estrategia preventiva, con la cual se evitarían ambientes tensos y emocionalmente insanos y los riesgos de descuido y fracaso académico (Salinas et al., 2002).

Sobre los roles asumidos en la situación de conflicto, los estudiantes enfatizaron en los surgidos en la relación entre pares Di Napoli (2012), en ellos destacan a quien busca ponerse del lado del amigo por lealtad o por la convicción en su 
capacidad para contribuir a una salida pacífica. En esta perspectiva pocas veces aludieron a los roles de los docentes y directivos como generadores o motivadores de la situación conflictiva, lo que no excluye el rol decisivo de estos como mediadores y agentes para la resolución.

Lamentablemente, desde la perspectiva de los participantes en la investigación, los docentes sólo toman conciencia de las situaciones de dificultad en las relaciones entre los estudiantes o se deciden a intervenirlas cuando por causas de éstas se pone de manifiesto la violencia, bien sea física o verbal, por esta razón es inaplazable para las instituciones educativas visibilizar el tema del conflicto y trabajar en perspectiva de su reconocimiento como fenómeno propio de las relaciones entre los diversos actores institucionales, máxime cuando los jóvenes concurren en indicar su existencia soterrada en lo cotidiano, sus posibilidades para transformarse en violencia con todos los riesgos que ello conlleva y sus alcances respecto a su relación con la trasgresión de la norma.

Lo anterior implica hacer lecturas constantes sobre situaciones como "la competitividad, la rivalidad y el abuso" (Ghiso y Ospina, 2010, p.546) y otras que puedan despertar el conflicto, pero igualmente avanzar desde la reflexión y la teoría a la acción deliberada, ya que es posible que el discurso acerca de la violencia, el conflicto y la convivencia escolares sea rico en construcciones escritas y probablemente también haya sido apropiado con elocuencia en los discursos del día a día, no obstante, y en términos de Magendzo (2017) al aludir a los contextos educativos para la paz:

No es suficiente hacer declaraciones y manifestaciones sobre la importancia que la paz tiene en el ámbito educativo si éstas no se reflejan en el quehacer integral de la misma, en un currículum, pedagogía, didáctica, en los sistemas de evaluación, en las relaciones interpersonales, en la cultura, en los reglamentos disciplinarios, en la convivencia pacífica, el proyecto institucional, en los niveles de participación donde todos los actores: estudiantes, docentes, autoridades, padres de familia, colaboradores, etc., tienen una voz y una postura pacífica para resolver la situaciones problemáticas que se afrontan a diario (p. 16 ).

Los actores institucionales están convocados al examen riguroso de sus actitudes y decisiones ante las circunstancias que tengan implícita la coexistencia del conflicto; la mirada intencionada y la atención inmediata, decidida y centrada en la autoridad son reclamo constante de los estudiantes, aún de aquellos que no se vinculan directamente con el mismo, pues sus alcances pueden llegar en cualquier momento a afectarlos y a comprometerlos.

La formación de la comunidad educativa para la resolución del conflicto puede también asumirse como un cometido fundamental, la preparación de los sujetos acompañada de entrenamiento en habilidades para evitarlo, sortearlo o intervenirlo bien pudiese incluso aminorar las circunstancias de violencia y favorecer significativamente los contextos para la convivencia.

Finalmente, en relación con la norma, es claro que los estudiantes tienen conocimiento de su valor y lugar para la regulación de la convivencia, pero su cumplimiento es causal de conflicto, lo que denota gran ambivalencia, ellos reconocen la norma como reguladora en pro del bien común, incluso como prevención del conflicto y la violencia, pero al observar sus acciones algunas contradicen este conocimiento, lo que motiva a pensar que sigue siendo necesaria la propuesta que desde hace casi dos décadas circula en los espacios 
educativos colombianos en relación con la construcción colectiva de la norma Míguez (2017), con la que se comprometa a los diversos actores en su ejecución y garantía de legitimidad.

Respecto a nuevas preguntas para futuras investigaciones relacionadas con el conflicto escolar bien pudiesen pensarse tópicos que orienten las miradas a la incidencia de la normatividad promulgada en Colombia en los últimos años sobre convivencia, al igual que sobre las estrategias dinamizadas en los centros educativos para hacer real los fundamentos teleológicos y prácticos de estas políticas, todo ello articulado en torno a la categoría conflicto.

De igual manera sería pertinente indagar a partir de un enfoque fenomenológico, es decir desde construcciones subjetivas, la relación entre el conflicto interior, o conflicto consigo mismo, y el conflicto escolar; esto a partir del planteamiento ofrecido en esta investigación por parte de dos jóvenes sustentado en la afirmación "para que haya un conflicto no necesariamente tiene que haber dos porque uno puede tener un conflicto con uno mismo, por eso no tiene que ser entre dos, por eso hay personas que se suicidan" (joven i).

Sobre el mismo no se profundizó, por cuanto los objetivos y las estrategias de recolección de información estaban orientadas a indagar exclusivamente acerca del conflicto escolar como situación de controversia entre dos o más personas; no obstante, se advierte este como un tema en pesquisas inherentes al conflicto en el ámbito escolar.

\section{Referencias}

Castillo, P. (2011). El acoso escolar: de las causas, origen y manifestaciones a la pregunta por el sentido que le otorgan los actores. Magis.
Revista Internacional de Investigación en Educación, 4 (8), pp. 415-428. Recuperado dehttp://revistas.javeriana.edu.co/index.php/ MAGIS/arti cle/view/3572doi: http://dx.doi. org/10.11144

Chaux, E. (2002). Buscando pistas para prevenir la violencia urbana en Colombia: conflictos y agresión entre niños y adolescentes. Revista de estudios sociales, (12), 43-53. Recuperado de https://revistas.uniandes.edu.co/ doi/pdf/10.7440/res12.2002.04 https://doi. org/10.7440/res53.2015.05

Concha-Eastman, A. y Krug, E. (2002). Informe mundial sobre la salud y la violencia de la OMS: una herramienta de trabajo. Revista panamericana de salud pública, 12(4), 227229. Recuperado de https://www.scielosp. org/pdf/rpsp/2002.v12n4/227-229/es\#top

Congreso de la República. Ley No. 1098. Diario Oficial República de Colombia, Bogotá, Colombia, 8 de noviembre de 2006 .

Coronel, A. G. y Puig de Stubrin, L. J. (2013). Violencia en las escuelas: políticas públicas y capacidades estatales de la provincia de santa fe (2003-2007), el caso de las oficinas responsables del acceso del problema a la agenda y la implementación de las políticas. Documentos y aportes en administración pública y gestión estatal, (21), 151-154. doi: https://doi.org/10.14409/da.v1i21.4154

Di Napoli, P. (2012). Jóvenes, violencia y escuela: un análisis de las relaciones entre grupos de pares en dos escuelas secundarias de Argentina. Revista Austral de Ciencias Sociales, (23), 25-45. doi: http://dx.doi.org/10.14482/ zp. 24.8727 
Foucault, M. (2002). Vigilar y castigar. Nacimiento de la prisión. Buenos Aires - Argentina. Editorial: Siglo XX.

Galeano Marín, M. E. (2004). Diseño de proyectos en la investigación cualitativa. Medellín Colombia. Editorial: Fondo Editorial Eafit.

Ghiso Cotos, A. M. y Ospina Otavo, V. Y. (2010). Naturalización de la intimidación entre escolares: un modo de construir lo social. Revista Latinoamericana de Ciencias Sociales, Niñez y Juventud,8, (1), 535-556. Recuperado de http://www.redalyc.org/articulo. oa? id=77315079025

López, V., Carrasco, C., Morales, M., Ayala, Á., López, J. y Karmy, M. (2011). Individualizando la Violencia Escolar: Análisis de Prácticas Discursivas en una Escuela $\mathrm{Mu}$ nicipal de la Región de Valparaíso. Psykhe, 20(2), 7-23. doi: http://dx.doi.org/10.4067/ S0718-22282011000200002

Luna Bernal, A. y De Gante-Casas, A. (2017). Empatía y gestión del conflicto en estudiantes de secundaria y bachillerato. Revista de educación y desarrollo, (40), 27-40. Recuperado de http://docplayer.es/63824774-Empatiay-gestion-de-conflictos-en-estudiantesde-secundaria-y-bachillerato.html

Magendzo, A. (2017). Reflexiones en torno a la paz que vive Colombia y su implicancia en la educación. Rutas de formación: Prácticas y Experiencias, 0(2), 15-17. doi: https://doi. org/10.24236/24631388.n2.2016.574

Míguez, D. P. (2017). Las complejidades de la convivencia ciudadana en la Escuela Media argentina. Un estudio de caso en la provincia de Buenos Aires. Poliphonía. Revista de Educación Inclusiva, 1, 23-45. Recuperado de https://revista.celei.cl/index. php/PREI/article/view/23-45/3

Ministerio Educación Nacional. Ley 1620. Diario Oficial República de Colombia, Bogotá, Colombia, 15 de marzo de 2013.

Ministerio Educación Nacional. Decreto Reglamentario 1965. Diario Oficial República de Colombia, Bogotá, Colombia, 11 de septiembre de 2013.

Olweus, D. (2004). Conductas de acoso y amenazas entre escolares. Madrid - España. Editorial: Morata.

Páramo, P. (2016). Investigación de estudio del caso: estrategia de indagación. En: P. Páramo. (Ed.). La investigación en Ciencias Sociales: estrategias de investigación. pp. 309-316. Bogotá - Colombia. Editorial: Departamento de publicaciones y comunicación gráfica de la Universidad Piloto de Colombia.

Ramírez-López, C. y Arcila-Rodríguez, W. (2014). Violencia, conflicto y agresividad en el escenario escolar. Educación y Educadores, 16(3), 411-429. Recuperado de: http://educacionyeducadores.unisabana.edu.co/index.php/eye/ article/view/2778/3350

Rincón Vásquez, J. C., y Barreto, I. (2016). Técnicas de muestreo para investigaciones sociales. En: P. Páramo. (Ed.). La investigación en Ciencias Sociales: estrategias de investigación. pp. 33-44. Bogotá Colombia. Editorial: Departamento de 
publicaciones y comunicación gráfica de la Universidad Piloto de Colombia.

Rodríguez Gómez, G., Gil Flores, J. y García Jiménez, E. (1996). Metodología de la investigación cualitativa. Granada - España. Editorial: Aljibe.

Salinas, S., Posada G. e Isaza M. (2016). A propósito del conflicto escolar. Revista Educación y Pedagogía, 14(34), 243-273. Recuperado de http://aprendeenlinea.udea.edu.co/ revistas/index.php/revistaeyp/article/ view/325110/20782596
Sandín Esteban, M. P. (2003). Investigación cualitativa y educación. Fundamentos y tradiciones. Madrid - España. Editorial: Mc Graw Hill.

Tijmes, C. (2012). Violencia y clima escolar en establecimientos educacionales en contextos de alta vulnerabilidad social de Santiago de Chile. Psykhe, 21(2), 105-117. doi: http:// dx.doi.org/10.7764/psykhe.21.2.548

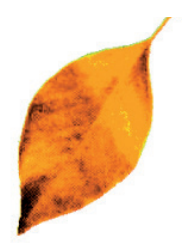

